\title{
The Emerging Field of RNA Nanotechnology
}

\author{
Peixuan Guo* \\ Nanobiomedical Center, College of Engineering and College of Medicine, University of Cincinnati, \\ Cincinnati, $\mathrm{OH} 45221$
}

\begin{abstract}
RNA can be designed and manipulated just like DNA while having different rules for base-pairing and displaying functions similar to proteins. The large variety of loops and motifs in RNA allow them to fold into numerous complicated structures. This diversity provides a platform for identifying viable building blocks for particle assemblies, substrate binding and manufacture engineering. RNA thermal stability allows production of multivalent nanostructures with defined stoichiometry. Here we review the unique qualities of RNA nanotechnology and their distinct properties inside the body. We describe techniques for constructing RNA nanoparticles from different building blocks and their applications in nanomedicine. Finally, we discuss challenges in predicting and synthesizing RNA and offer some perspectives on the yield and cost of RNA production.
\end{abstract}

Nanotechnology addresses the creation and application of materials at the nanometre scale using either top-down approaches or bottom-up assembly. Macromolecules of DNA, RNA and proteins have intrinsically defined features at the nanoscale and may serve as powerful building blocks for bottom-up fabrication of nanostructures and nanodevices. A pioneering concept proposed by Ned Seeman 30 years ago has led to the development of the field known as DNA nanotechnology ${ }^{1-3}$. Peptides or proteins have also been studied extensively for applications in nanotechnology $4-7$. The concept of RNA nanotechnology has been around for more than a decade ${ }^{8-13}$ (for review, see ref ${ }^{14,15}$ ) and the first evidence for the construction of RNA nanoparticles through self-assembly of multiple reengineered natural RNA molecules was reported in 1998 (ref. ${ }^{8}$ ). However, RNA nanotechnology has only recently become increasingly popular due to the recognition of its potential in the treatment of cancer, viral infection, genetics diseases and other applications in nanomedicine (Fig. 1).

\section{Significance and uniqueness of RNA Nanotechnology}

RNA can be designed and manipulated with a level of simplicity characteristic of DNA, meanwhile displaying flexibility in structure and diversity in function similar to that of proteins, including enzymatic activities. Although RNA nanotechnology can be regarded as a subdivision of DNA nanotechnology, the uniqueness of RNA properties distinct from those of DNA might warrant a new emerging discipline.

RNA is a polymer made up of four different nucleotides: adenine, cytosine, guanine and uridine. RNA possesses not only Watson-Crick base pairing but also noncanonical base pairing which promotes folding into rigid structural motifs distinct from the structure of single-stranded DNA. The noncanonical property facilitates loop-receptor interactions and

Correspondence and requests for materials should be addressed to: Peixuan Guo, Vontz Center for Molecular Studies, Room 2308, ML\#0508, 3125 Eden Avenue, University of Cincinnati, Cincinnati, OH 45267, USA, Phone: (513)558-0041, guopn@ucmail.uc.edu, guop@purdue.edu.

Additional information Reprints and permission information is available online at http://npg.nature.com/reprintsandpermissions/. 
allows the creation of synthetic ribozymes ${ }^{16,17}$. It also allows the formation of special structural motifs ${ }^{18-20}$. Tertiary interactions mediate GNRA/receptor interactions ${ }^{10}$, and formation of micrometer sized RNA filaments ${ }^{10,12,21-24}$ (Fig. 2g,h,i,j). Currently, an RNA up to 80 nucleotides can be synthesized commercially. An 80-nucleotide RNA can display up to $10^{48}\left(4^{80}\right)$ different structures with unique shapes involving non-canonical interactions. Such a huge pool of rich structural conformations would ease the search for viable partners in particle assemblies, substrate binding, building architectures, and manufacture engineering.

Typically, RNA contains a large variety of single-stranded stem-loops for intra- and/or intermolecular interactions. These loops can serve as mounting dovetails, and thus external linking dowels might not be needed for nanomachine fabrication and assembly. Loops and motifs also allow for construction of a more complicated secondary structure. Versatility and low-energy folding delivers a significant ad vantage. Furthermore, RNA molecules can possess special functionalities such as aptamer, riboswitch, ribozyme, and siRNA.

The RNA/RNA double helix is the most stable among the three helices: RNA/RNA, RNA/ DNA and DNA/DNA ${ }^{25,26}$. RNA motifs and modules with special bends or stacks are particularly stable. The thermodynamic stability has been defined as the free energy required for complex formation, or in some cases, to unwind the helix $\left(\Delta \mathrm{G}^{0}=-\mathrm{G}^{0}\right.$ helix $=\mathrm{G}^{0}{ }_{\text {unwind }}$ ); thus, the lower the free energy $\left(-\mathrm{G}^{0}\right.$ helix $)$ the complex holds, the more stable the complex. $\Delta \mathrm{G}^{0}$ is affected by neighboring sequences. Based on the nearest-neighbor model, $-\mathrm{G}^{0}{ }_{\text {helix }}$ has been calculated to be lower for RNA than DNA ${ }^{25,26}$. The hydrogen bonding and phosphodiester backbone of RNA and DNA helix are very similar, and the difference in energy between DNA dA:dT and RNA A:U base pairs are trivial (Table 1). However, under physiological conditions, the RNA helix displays A-type configuration whereas the DNA helix is predominantly B-type. The 2'-OH in RNA ribose locks the ribose into a 3'-endo chair conformation which does not favor a B-helix. Base stacking is governed by vander Waals interaction, which contributes directly to the enthalpy. Though the difference in the stacking interaction is small between DNA and RNA, the sum over multiple base pairs can make a difference in the helix stability. Thus, RNA nanoparticles are more stable thermodynamically than their DNA counterparts. DNA tiles can assemble through sticky ends of four to six nucleotides. Surely, four to six nucleotides of RNA are sufficient to produce stable RNA helices in solution ${ }^{12}$. In certain cases, as few as two nucleotides can promote complex formations in $\mathrm{RNA}^{27-31}$.

\section{Distinct attributes of RNA inside the body}

Cell endosome escape is an important consideration for in vivo delivery. Therapeutic particles are initially recognized by cell surface receptor(s) and then delivered through entry into the cellular endosome. The $\mathrm{pH}$ within the endosome ranges from 4.3 to $5.8^{32}$, where RNA is more stable than DNA (Table 1). In acidic environments ${ }^{33}$, the protonation of DNA purine bases leads to depurination and the resulting apurinic DNA is susceptible to cleavage. The higher stability of RNA in acid ic environments is especially useful in therapy since RNA will survive in the endosome after cell entry, and disperse throughout the cell during endocytosis.

Another intriguing property of RNA is the possibility to produce self-assembled RNA nanoparticles in vivo. In contrast to DNA, small RNA molecules are transcribed in the cell using DNA as a template. By using an inducible promoter ${ }^{34}$ and appropriate terminators for transcription, small RNA molecules can be controllably produced. RNA can be processed into the desired length by including delta ribozymes at both the upstream and downstream terminals for cis-cleavage ${ }^{35}$. Natural RNA nanoparticles such as dimers ${ }^{36-39}$ and 
hexamers ${ }^{8,9}$ have been discovered in cells. Sequences, such as pRNA ${ }^{35}$ or tRNA ${ }^{40,41}$ for guiding the self- assembly of RNA nanoparticles with functionalities such as siRNA ${ }^{42,43}$, ribozyme ${ }^{35}$, or aptamer ${ }^{40}$ can be incorporated within the DNA template in vivo ${ }^{35}$.

Small RNAs, such as riboswitch, with regulatory functions ${ }^{44-47}$ within the cell may be viewed as Boolean networks based on logic operations ${ }^{48,49}$. Input nodes can be viewed as RNA nanostructures and the output, e.g., the activation of a pathway, is based upon logic functions of input RNA concentrations. Multiple small RNA regulators can be used to regulate the in vivo products and functional pathways, with controls by induct ion or repression through the trans- and cis-actions. Varieties of small RNA can work cooperatively, synergistically, or antagonistically based on the design to produce computational logic circuits as conjunctive or disjunctive normal forms or other kind of logic operation. By designing the logic network of AND/NOT/OR different gates in the cell, an "RNA computer" can be theoretically implemented and applied to bacterial, yeast, and mammalian systems ${ }^{48,49}$.

\section{Techniques for constructing RNA nanoparticles}

Construction of nanoparticles requires the use of programmable, addressable and predictable building blocks. Self-assembly of RNA building blocks in a predefined manner to form larger two-, three-, and four-dimensional structures is a prominent bottom-up approach and represents an important means by which biological techniques and biomacromolecules can be successfully integrated into nanotechnology $12,50,51$.

Within the realm of self-assembly there are two main subcategories: templated and nontemplated assembly. Templated assembly involves the interaction of RNAs with one another under the influence of a specific external force, structure, or spatial constraint. RNA transcription, hybridization, replication, molding, and phi29 pRNA hexameric ring formation are all within this category. Non-templated assembly involves the formation of a larger structure by individual components without any external influence. Examples include, ligation, chemical conjugation, covalent linkages, loop/loop interactions of RNA such as the HIV kissing loop, and phi29 pRNA dimer or trimer formation ${ }^{10,12,50-52}$. Various approaches available for RNA nanoparticle construction are discussed below.

The first approach utilizes the assembly mechanism of natural RNA nanoparticles that can form unique and intriguing multimers in vivo. For example, the retrovirus kissing loops facilitate genomic RNA dimerization ${ }^{36,37}$. The pRNA of the bacteriophage phi29 DNA packaging motor assembles into dimers and hexamers via hand-in-hand interactions between two right and left interlocking loops $8,12,28,39,52,53$. The bicoid mRNA of Drosophila embryos forms dimers via hand-in-arm interactions ${ }^{38}$. E. coli noncoding RNA DsrA assembles into stripe patterns via their built-in palindrome sequence ${ }^{23}$. The assemblies of RNA nanoparticlesin vitro that mimic their natural counterparts were reported twelve years $\mathrm{ago}^{8}$. The novel HIV kissing loop mechanism has also inspired the design of tecto-RNA architectures ${ }^{13,29}$.

The Second tactic is to import the well-developed principle of DNA nanotechnology into the RNA field. While RNA is unique, certain common features between RNA and DNA in structure and chemistry make it compulsory for the developing RNA nanotechnology field to draw experiences from the well-developed DNA nanotechnology wealth and apply some, not all, of the approaches in DNA to the RNA field.

DNA nanotechnology utilizes the nature of DNA complimentarity for the construction of nanomaterials via intermolecular interactions of DNA strands. A variety of elegant shapes have been created with precise control over their geometries, periodicities and topologies 
(Fig. 2) (see recent reviews ${ }^{1-3}$ and the references therein). Various cross-over motifs have been designed via reciprocal exchange of DNA backbones ${ }^{3}$. Branched DNA tiles have been constructed using sticky ends and cross-over junction motifs, such as tensegrity triangles (rigid structures in perioidic array form) ${ }^{54}$, and algorithmic self-assembled Sierpinski triangles (aperiodic arrays of fractal patterns) ${ }^{55}$. The DNA tiles can further self-assemble into nanotubes, helix bundles ${ }^{56}$ as well as complex DNA motifs and arrays for positioning nanoparticles, proteins or dyes with precise control, such as polycatenated DNA ladders ${ }^{57}$. Elegant 3D DNA networks using a minimal set of DNA strands with topologies such as cubes, polyhedrons, prisms, and buckyballs have also been fabricated based on junction flexibility and edge rigidity ${ }^{3,58}$. A continuous growth of the tensegrity triangle within the periodic DNA module has resulted in the formation of DNA crystals diffracting to $4 \AA$ resolution ${ }^{59}$.

A striking marvel demonstrating the addressable and programmable property of DNA is Rothemund's DNA origami ${ }^{60}$, where a long single-stranded viral DNA is used as a scaffold for binding shorter strands to generate well-defined 2D and 3D configurations. DNA origami was subsequently applied to build 3D boxes that can be locked and unlocked ${ }^{61}$, nanoarrays for label-free detection of substrates ${ }^{62}$ and for structure elucidation of organized proteins ${ }^{63}$. Rationally designed supramolecular DNA assemblies can be conjugated with organic and inorganic molecules, such as conjugation of porphyrins on parallel DNA helix bundles ${ }^{64}$ nanomagnets ${ }^{65}$ and elegant nanomachines ${ }^{58,66}$. Replicable DNA architectures have been achieved to scale-up DNA nanostructure production for practical applications by using enzymatic rolling circle replication, bacterial cells infected with a viral vector ${ }^{67}$, or chemical approaches for amplifying branched DNA arms ${ }^{68}$.

Although the folding properties of RNA and DNA are not exactly the same, the fundamental principles in DNA nanotechnology are applicable to RNA nanotechnology. The use of 3WJ (three-way junction) and 4WJ 18,29 to build novel and diverse RNA architectures are very similar to the branching approaches in DNA ${ }^{1,3}$ (Fig.2a,b,e,f). The formation of Jigsaw puzzles was demonstrated in both RNA ${ }^{13}$ and DNA ${ }^{69}$ (Fig.2a,b). Both RNA ${ }^{12,23,30}$ and DNA ${ }^{70}$ polymers can be developed into bundles by combining elongation and expansion in the $\mathrm{x}$-y directions (Fig.2g,h,i,j). The finding that insertion of bulges in RNA helix leads to the formation of twisted bundles ${ }^{12}$ (Fig.2i) was later demonstrated in DNA (Fig.2g), revealing that insertions and deletions of bases can lead to the formation of twisted DNA bundles with handedness ${ }^{70}$ thereby illustrating the same basic principle. However, RNA is more rigid in bulge structure due to non-canonical interactions, while in DNA, the twisting requires the interaction of two DNA helixes with four strands ${ }^{70}$.

Recently, RNA cubic scaffolds ${ }^{71}$ were constructed using several RNA sequences that do not fold on themselves but self-assemble with one another in a defined manner. This strategy is reminiscent of DNA nanotechnology, but in contrast to DNA strategies, RNA synthesis can be coupled to RNA self-assembly to generate fully assembled RNA cubes during in vitro transcription.

The third tactic is to apply computational methods in RNA nanoparticle construction. Computational approaches can be used to guide the design of novel RNA assemblies and to optimize sequence requirements for the production of nanoscale fabrics with controlled direction and geometry $37,72-75$. In contrast to traditional methods in which raw materials are selected rather than designed for a given application, the next generation of building blocks can be designed a priori for programmed assembly and synthesis. There are two steps in building RNA nanoparticles. The first is a computational approach (e.g. using Kinefold ${ }^{72}$ ) utilizing the spontaneous self-folding property of RNA into defined structures via base/base interactions based on their characteristic $\Delta \mathrm{G}^{76}$. The second is the spontaneous assembly of 
the resulting RNA building blocks into larger assemblies based on the predicted architecture. This creates an effective computational pipeline for generating molecular models of RNA nanostructures. A recent example is the construction of cubic RNA-based scaffolds, whereby RNA sequence designs were optimized to avoid kinetic traps ${ }^{77}$.

The fourth tactic is to utilize the existing RNA structure, or with known function, as building blocks in RNA nanoparticle construction. The structure of RNA motifs and mechanisms of RNA folding and sequence interactions have been investigated for many years. Varieties of mechanisms in RNA loop/loop interactions $8,12,31$, tertiary architecture contacts ${ }^{12,15,30}$, and formation of special motifs $12,21,29-31,78-82$ have been elucidated. Building-blocks are first synthesized after computing intra- and inter-molecular folding. Nanoparticles are built via spontaneous templated or nontemplated self-assembly as planned. A rich resource of welldeveloped databases can be utilized to extract known RNA structural units for construction of novel RNA nanoparticles with desired properties ${ }^{37,83,84}$.

Several methods have been pursued for RNA nanoparticle construction borrowing RNA properties in loop/loop interactions. The first method is based on the structural features of the pRNA of the bacteriophage phi29 DNA packaging motor ${ }^{8,85}$ which uses a hexameric RNA ring to gear the machine $28,86,87$. The pRNA has been reengineered to form dimers, trimers, tetramers, hexamers and arrays via hand-in-hand or foot-to-foot interactions between two interlocking loops 12,52 (Fig. 3). Dimers are formed using two building blocks with $\mathrm{A} / \mathrm{b}^{\prime}$ (right and left hand, respectively) and $\mathrm{B} / \mathrm{a}^{\prime}$ (Fig. 3). Trimers are formed using three building blocks with $\mathrm{A} / \mathrm{b}^{\prime}, \mathrm{B} / \mathrm{c}^{\prime}$, and $\mathrm{C} / \mathrm{a}^{\prime 12,50,51}$. Dimers of an extended configuration (twins) can also be efficiently self-assembled by introducing a palindrome sequence into the $3^{\prime}$-end of the pRNA ${ }^{12}$. These nanoparticles have been used successfully as polyvalent vehicles to deliver a variety of therapeutic molecules (Fig.3f-j) (see Section 4) ${ }^{12,52}$. The use of pRNA as building blocks for the construction of RNA arrays has also been achieved ${ }^{12}$. When three twins, $\mathrm{Ab}^{\prime}, \mathrm{Bc}^{\prime}$ and $\mathrm{Ca}^{\prime}$ are mixed, loop/loop interlocking makes the particles grow in threedimensions.

The second method is the RNA "architectonics"13, whereby structural modules specifying for bends or stack can be encoded within artificial RNA sequences for self-assembling higher order specific shapes of RNA. Examples include, RNA filaments ${ }^{10,21,24}$ (Fig.2j), molecular jigsaw puzzle units called tectosquares ${ }^{13,29}$ (Fig.2f) and tRNA antiprisms ${ }^{88}$.

The third method is the application of $3 \mathrm{WJ}$ (three way junction) and $4 \mathrm{WJ}$ that are selected from known RNA structures or motifs ${ }^{18,19}$ to serve as the cornerstone in nanoparticle construction (Fig.2 ${ }^{29,76}$. Some examples include: RNA-structural motif (from rRNA) to guide the tetramer assembly of L-shaped tecto RNAs; 3WJ-motif (from 23S rRNA) to construct T-shaped arrangement of three helices; and, tRNA motifs consisting of 4- and 5WJ to fold L-shaped tertiary structures ${ }^{29,37}$.

The fourth method is to assemble non-natural functional RNAs with defined 3D structures using synthetic ribozyme ligase by employing molecular design of RNA based on the in vitro selection technique ${ }^{16,17}$. Conformational switch of RNA nanostructures can also be constructed using a peptide-binding RNA structural motif ${ }^{20}$.

The fifth method is the use of a palindrome sequence, which differs from the sticky end, at the 5' or 3' end of the RNA. The molecule will spontaneously assemble via self annealing of the palindrome sequence immediately after in vitro transcription or chemical synthesis, before purification ${ }^{12}$. This method is useful for the creation of bundles, especially for designing 3D branches. Since each 11-nucleotides of the A-form RNA generates one helical turn of $360^{\circ}$, the angle or the direction for RNA fiber extension is controllable by varying the number of nucleotides in the helix containing the palindrome sequence. 


\section{Applications of RNA Nanotechnology}

The versatility of RNA structure, the low free energy in RNA annealing, the amenability in sequence, the options in structure control, and the property of self-assembly make RNA an ideal material in nanotechnology applications. It is possible to adapt RNA to construct ordered, patterned, or preprogrammed arrays or superstructures (Fig.2h,i). RNA sequences can mediate the growth of hexagonal palladium nanoparticles ${ }^{89}$; programmable selfassembling property of RNA ladders can direct the arrangement of cationic gold nanoparticles; periodically spaced RNA architectures can serve as a scaffold for nanocrowns ${ }^{90}$. Geometrically symmetrical shapes such as dimers, trimers, or polygons can be constructed from RNA 12,13,52. As the symmetrical shapes facilitate the formation of crystals, RNA might serve as scaffolds for X-ray crystallography. In addition, self-assembly interaction between interlocking loops, self-linkages via a palindrome sequence, the continued growth into a hierarchical structure, and ease in conjugation and biocompatibility make RNA a good candidat e for construction of scaffolds for tissue engineering 12,21,23. Several laboratories have developed RNA aptamers as biosensors ${ }^{91}$.

RNA's novel roles in nanomedicine application include cell recognition and binding for diagnosis ${ }^{92}$; targeted delivery via receptor mediated endocytosis ${ }^{93}$; intracellular control and computation via gene silencing and regulation ${ }^{48,49}$, nuclear membrane penetration, and brain blood barrier passing ${ }^{94}$. The most important therapeutic RNA moieties are discussed below.

Small interfering $\boldsymbol{R N} \boldsymbol{A}^{42,43}$ (siRNA) is a helix with 20-25 nucleotides that interferes with gene expression through the cleavage of mRNA by a protein/RNA complex named RISC (RNA-induced silencing complex). The siRNA specifically suppresses the expression of a target protein whose mRNA includes a sequence identical to the sense strand of the siRNA. The discovery led to the award of the 2006 Nobel Prize to Andrew Fire and Craig Mello ${ }^{42}$.

Ribozyme $^{95,96}$ is an RNA molecule that has enzymatic activity. They have significant therapeutic potentials capable of regulating gene function by intercepting and cleaving RNA substrates, such as mRNA or the viral genome of RNA containing a sequence complementary to the catalytic center of the ribozyme. The discovery also led to the award of the 1989 Nobel Prize to Thomas Cech and Sydney Altman.

RNA aptamer ${ }^{97,98}$ is a family of oligonucleotides with functions similar to that of antibodies in their ability to recognize specific ligands (organic compounds, nucleotides, or peptides) through the formation of binding pockets ${ }^{92}$. SELEX ${ }^{99}$ is the method to screen for the aptamers from randomized RNA pools developed in vitro by Ellington and Szostak ${ }^{97}$, and Tuerk and Gold ${ }^{98}$. Using this technique, various aptamers have been selected for targeting markers relevant to diseases ${ }^{92,100,101}$.

Riboswitches ${ }^{102}$ are RNA components that bind small molecules and control gene expression in response to an organism's needs. As a biological control mechanism, riboswitches can recognize metabolites, induce premature termination of mRNA transcription, block ribosomes from translating mRNAs, cleave mRNAs, and even trigger mRNA destruction. Therefore, RNA switches can be reengineered to create a new generation of controllers regulated by drug-like molecules to tune the expression levels of targeted genes in vivo. Such RNA-based gene-control machines hold promise in future gene therapies by supplying nanoscale cis-acting modulation ${ }^{103,104}$.

Various RNA moieties including siRNAs, ribozymes, antisense RNAs, aptamers, riboswitches, as well as other catalytic or editing RNAs can be easily fused o $r$ conjugated into RNA nanoparticles (Fig.3f-j). The advantage of RNA nanomedicine includes: 1) self- 
assembly (see Section 2 for self-assembly and self-processing in vivo); 2) multi-valency; 3) targeted delivery; 4) protein-free; 5) nanoscale size; 6) controlled synthesis with defined structure and stoichiometry; 7) combining therapy and detection of therapy effects into one particle.

Bottom-up assembly of RNA can lead to multi-valency ${ }^{51}$. Each subunit may be separately functionalized to carry different therapeutic payloads, reporters and/or targeting ligands (Fig.2d, Fig.3f-j). Cell-type-specific delivery allows a lower concentration of the drug to be administered, thus reducing the side effects. The multivalent approach is similar to that of cocktail therapy, in which a mixture of drugs is used to produce a synergistic effect. The multi-valency offers an additional unique advantage in that therapy and detection of therapeutic effects may be combined into one nanoparticle conducted under a single administration ${ }^{12,50,51}$.

Currently, a variety of other polyvalent nanoparticles have been developed; however, producing homologous particles and consistent reproduction of copy numbers within the population is challenging. Any uncertainty in structure and stoichiometry could cause unpredictable side effects or nonspecific toxicity. Using RNA nanotechnology, the production of homogeneous nanoparticles can be "manufactured" with high reproducibility, and defined structure and stoichiometry, thus facilitating quality and safety control.

The size of RNA particles in the nanometer-scale is another advantage. For effective delivery to diseased tissues, many studies suggest that particles ranging from 10-50 nm are optimal for a nonviral vector as they are large enough to be retained by the body yet small enough to pass through the cell membrane via the cell surface receptor mediated endocytosis ${ }^{105}$. Nanoparticle delivery has the potential to improve the pharmacokinetics, pharmacodynamics, biodistribution, and safety of this newly emerging modality.

The protein-free nature will avoid the induction of antibodies, thus allowing repeated administration for treatment of chronic diseases including cancers, viral infections, and genetic ailments. In addition, RNA nanoparticles are classified by the FDA as chemical rather than biological entities and this classification will speed-up the FDA approval.

The feasibility of RNA nanotechnology in disease therapy has been exemplified in the phi29 pRNA therapeutic system $14,35,50,51,106,107$. Incubation of the synthetic polyvalent RNA nanoparticles containing receptor-binding aptamers or ligands resulted in cell binding and entry of the incorporated therapeutics, subsequently modulating apoptosis ${ }^{50,51}$. The delivery efficiency and therapeutic effect were later confirmed in animal trials ${ }^{50,51}$. The 3D design, circular permutation, folding energy alteration, and nucleotide modification of RNA were applied to generate RNase resistant RNA nanoparticles with low toxicity and to ensure processing of the chimeric RNA complexes into siRNA by Dicer after delivery.

\section{Challenges and perspectives}

RNA nanoparticle construction involves conjugation of functionalities, crosslinking of modules, labelling subunits, and chemical modification of nucleotides. Methods in synthesizing RNA building blocks include both chemical and enzymatic approaches. While great progress has been made, improvements are in demand.

Prediction of RNA structure or folding for particle assembly remains a great challenge. Due to the unusual folding properties such as non-canonical base pairing, the rules that elucidate RNA folding are yet to be sorted out. Currently, using the RNA 2D prediction program by Zuker, typically only $70 \%$ of the $2 \mathrm{D}$ folding prediction is accurate based on experimental data ${ }^{74,75}$. Clearly, predicting the RNA 3D and 4D structures is even more elusive. 
Computer-aided programs in RNA structure prediction remain to be explored. Programs for the computation of inter- molecular interactions of RNA subunits for quaternary nanostructure formation are in imperative demand.

Natural RNA is sensitive to RNase and is especially unstable in serum or in the body. RNA's instability has long hindered its application as a construction material. Improvement of RNA stability is greatly important and is progressing rapidly, including chemical modification of the base (e.g. 5-Br-Ura and 5-I-Ura); phosphate linkage (e.g. phosphothioate, boranophosphate); and/or the C2' (e.g. 2'-fluorine, 2'-O-methyl or 2'amine $)^{108}$. Other attempts include Peptide Nucleic Acids, Locked Nucleic Acids and their respective derivatives PolyCarbamate Nucleic Acids ${ }^{109}$ or Locked Nucleic Acids with a bridge at different positions $\left(2^{\prime}-4^{\prime}, 1^{\prime}-3^{\prime}\right){ }^{110}$. The $3^{\prime}$-end capping also improved the base pairing selectivity in duplex formation ${ }^{111}$. For all these methods, the $2^{\prime} \mathrm{F}$-modification is the most appraisable since it has minimal detrimental effect on RNA folding and function.

Loop/loop interaction is one of the unique approaches to assemble quaternary RNA nanoparticles; however, dissociation of loops can occur when the concentration is reduced. Crosslinking agents, such as psoralen, nitrogen mustard derivatives, and transition metal compounds ${ }^{112}$ can promote the formation of stable RNA complexes. Recent advancements include various bifunctional agents separated by linkers and phenolic derivatives ${ }^{113}$ to increase the efficiency of crosslinking. Long-range $(>9 \AA)$ and short range $(1.5 \AA)$ photoaffinity crosslinking can be achieved using azidophenacyl derivatives and thionucleosides, such as 6-thioguanosine and 4-thiouridine, respectively.

For fluorescent labelling, single conjugation of fluorophores at the $5^{\prime}$ - or $3^{\prime}$-end is preferable to prevent physical hindrance. End-labelling is not difficult with chemical synthesis of small RNA, however, it is challenging for long RNA requiring enzymatic methods. To meet this challenge, GMP or AMP derivative s that can only be used for transcription initiation, but not for chain elongation, have been used. Fluorescent RNA can also be easily synthesized in vitro with T7 RNA polymerase using a new a gent $\mathrm{tCTP}{ }^{114}$.

The challenges of in vivo computation using RNA ${ }^{48,49}$ include scaling the logic operations with a large number of inputs, extending input signal types, and nonspecific actions resulting in targeting unexpected or undesired pathways.

The results of modification related to RNA folding and in vivo toxicity of the nucleotide derivatives remain to be explored. Due to the metabolism and biocompatibility issues, the most stable RNA might not necessarily be the most desirable; retention of particles within an appropriate time period is more attractive.

The most challenging aspect of RNA therapeutics is the yield and cost of RNA production. Commercial RNA chemical synthesis can only offer 40 (conservative) to 80 (with low yield) nucleotides. Acetalester 2'-OH protecting groups, such as pivaloyloxymethyl, have been reported to enhance chemical synthesis of RNA. RNase ligase II has been shown to be a good alternative over the traditional T4 DNA ligase to generate longer RNA by ligation of two shorter synthetic RNA fragments ${ }^{115}$. In enzymatic synthesis, heterogeneity of the 3 '-end has been an issue ${ }^{116}$; this can be addressed by extending the transcribed sequence beyond $t$ he intended end and then cleaving the RNA at the desired site using ribozymes, DNAzymes, or RNase $\mathrm{H}^{115-117}$. Large scale RNA complexes produced in bacteria escorted by a t RNA vector have also been reported ${ }^{40,41}$. Based on the rapid reduction of cost over the history of DNA synthesis, it is expected that the cost of RNA synthesis will gradually decrease with the development of industrial-scale RNA production technologies. 
In conclusion, natural or synthetic RNA molecules can fold into pre-defined structures that can spontaneously assemble into nanoparticles with multiple functionalities. The field of RNA nanotechnology is emerging but will play an increasingly important role in medicine, biotechnology, synthetic biology and nanotechnology.

\section{Acknowledgments}

We thank John Rossi, Peter Stockley, Andrew Ellington, Shane Fimbel, Jason Lu, Farzin Haque, Anne Vonderheide, Randall Reif, Chaoping Chen, Mathieu Cinier and Feng Xiao for insightful comments; Chad Schwartz, Yi Shu, Jia Geng, for their assistance in preparation of this manuscript. The work in the author's laborat ory is supported by NIH grants GM059944, EB003730, and NIH Nanomedicine Development Center entitled "Phi29 DNA Packaging Motor for Nanomedicine" (PN2 EY018 230) through the NIH Roadmap for Medical Research, as well as contract from Kylin Therapeutics, Inc., of which the author is a cofounder.

\section{References}

1. Lin C, Liu Y, Yan H. Designer DNA nanoarchitectures. Biochemistry. 2009; 48:1663-1674. [PubMed: 19199428]

2. Aldaye FA, Palmer AL, Sleiman HF. Assembling materials with DNA as the guide. Science. 2008; 321:1795-1799. [PubMed: 18818351]

3. Seeman NC. Nanomaterials based on DNA. Annu Rev Biochem. 2010; 79:65-87. [PubMed: 20222824]

4. Moll D, et al. S-layer-streptavidin fusion proteins as template for nanopatterned D. molecular arrays. Proc Natl Acad Sci USA. 2002; 99:14646-14651. [PubMed: 12417763]

5. Cui H, Muraoka T, Cheetham AG, Stupp SI. Self-assembly of giant peptide nanobelts. Nano Lett. 2009; 9:945-951. [PubMed: 19193022]

6. Adler-Abramovich L, et al. Self-assembled arrays of peptide nanotubes by vapour deposition. Nature Nanotechnology. 2009; 4:849-854.

7. Knowles TP, et al. Nanostructured films from hierarchical self-assembly of amyloidogenic proteins. Nat Nanotechnol. 2010; 5:204-207. [PubMed: 20190750]

8. Guo P, et al. Inter-RNA interaction of phage phi29 pRNA to form a hexameric complex for viral DNA transportation. Mol Cell. 1998; 2:149-155. [PubMed: 9702202]

9. Zhang F, et al. Function of hexameric RNA in packaging of bacteriophage phi29 DNA in vitro. Mol Cell. 1998; 2:141-147. [PubMed: 9702201]

10. Jaeger L, Leontis NB. Tecto-RNA: One dimensional self-assembly through tertiary interactions. Angew Chem Int Ed Engl. 2000; 39:2521-2524. [PubMed: 10941124]

11. Jaeger L, Westhof E, Leontis NB. TectoRNA: modular assembly units for the construction of RNA nano-objects. Nucleic Acids Res. 2001; 29:455-463. [PubMed: 11139616]

12. Shu D, et al. Bottom-up assembly of RNA arrays and superstructures as potential parts in nanotechnology. Nano Lett. 2004; 4:1717-1723. [PubMed: 21171616]

13. Chworos A, et al. Building programmable jigsaw puzzles with RNA. Science. 2004; 306:20682072. [PubMed: 15604402]

14. Guo P. RNA Nanote chnology: Engineering, Assembly and Applications in Detection, Gene Delivery and Therapy. Journal of Nanoscience and Nanotechnology. 2005; 5(12):1964-1982. [PubMed: 16430131]

15. Jaeger L, Chworos A. The architectonics of programmable RNA and DNA nanostructures. Curr Opin Struct Biol. 2006; 16:531-543. [PubMed: 16843653]

16. Ikawa Y, Tsuda K, Matsumura S, Inoue T. Denovo synthesis and development of an RNA enzyme. Proc Natl Acad Sci U S A. 2004; 101:13750-13755. [PubMed: 15365187]

17. Matsumura S, et al. Coordinated control of a designed trans-acting ligase ribozyme by a loopreceptor interaction. FEBS Lett. 2009; 583:2819-2826. [PubMed: 19631647]

18. Leontis NB, Lescoute A, Westhof E. The building blocks and motifs of RNA architecture. Curr Opin Struct Biol. 2006; 16:279-287. [PubMed: 16713707] 
19. Schroeder KT, McPhee SA, Ouellet J, Lilley DM. A structural database for k-turn motifs in RNA. RNA. 2010; 16:1463-1468. [PubMed: 20562215]

20. Li X, Horiya S, Harada K. An efficient thermally induced RNA conformational switch as a framework for the functionalization of RNA nanostructures. J Am Chem Soc. 2006; 128:40354040. [PubMed: 16551112]

21. Nasalean L, Baudrey S, Leontis NB, Jaeger L. Controlling RNA self-assembly to form filaments. Nucleic Acids Res. 2006; 34:1381-1392. [PubMed: 16522648]

22. Liu B, Baudrey S, Jaeger L, Bazan GC. Characterization of tectoRNA assembly with cationic conjugated polymers. J Am Chem Soc. 2004; 126:4076-4077. [PubMed: 15053575]

23. Cayrol B, et al. A nanostructure made of a bacterial noncoding RNA. J Am Chem Soc. 2009; 131:17270-17276. [PubMed: 19821568]

24. Geary C, Chworos A, Jaeger L. Promoting RNA helical stacking via A-minor junctions. Nucleic Acids Res. 2010

25. Sugimoto N, et al. Thermodynamic parameters to predict stability of RNA/DNA hybrid duplexes. Biochemistry. 1995; 34:11211-11216. [PubMed: 7545436]

26. Searle MS, Williams DH. On the stability of nucleic acid structures in solution: enthalpy-entropy compensations, internal rotations and reversibility. Nucleic Acids Res. 1993; 21:2051-2056. [PubMed: 7684832]

27. Kitamura A, et al. Analysis of intermolecular base pair formation of prohead RNA of the phage phi29 DNA packaging motor using NMR spectroscopy. Nucleic Acids Res. 2008; 36:839-848. [PubMed: 18084020]

28. Chen C, Zhang C, Guo P. Sequence requirement for hand-in-hand interaction in formation of pRNA dimers and hexamers to gear phi29 DNA translocation motor. RNA. 1999; 5:805-818. [PubMed: 10376879]

29. Severcan I, et al. Square-shaped RNA particles from different RNAf olds. Nano Lett. 2009; 9:1270-1277. [PubMed: 19239258]

30. Severcan I, et al. A polyhedron made of tRNAs. Nat Chem. 2010; 2:772-779. [PubMed: 20729899]

31. Hansma HG, Oroudjev E, Baudrey S, Jaeger L. TectoRNA and 'kissing-loop' RNA: atomic force microscopy of self-assembling RNA structures. J Microsc. 2003; 212:273-279. [PubMed: 14629553]

32. Lee RJ, Wang S, Low PS. Measurement of endosome $\mathrm{pH}$ following folate receptor-mediated endocytosis. Biochim Biophys Acta. 1996; 1312:237-242. [PubMed: 8703993]

33. Pogocki D, Schoneich C. Chemical stability of nucleic acid-derived drugs. J Pharm Sci. 2000; 89:443-456. [PubMed: 10737906]

34. Laurenti E, et al. Inducible gene and shRNA expression in resident hematopoietic stem cells in vivo. Stem Cells. 2010; 28:1390-1398. [PubMed: 20641037]

35. Hoeprich S, et al. Bacterial virus phi29 pRNA as a hammerhead ribozyme escort to destroy hepatitis B virus. Gene Ther. 2003; 10:1258-1267. [PubMed: 12858191]

36. Chang KY, Tinoco I Jr. Characterization of a "kissing" hairpin complex derived from the human immunodeficiency virus genome. Proc Natl Acad Sci U S A. 1994; 91(18):8705-8709. [PubMed: 8078946]

37. Bindewald E, et al. RNAJunction: a database of RNA junctions and kissing loops for threedimensional structural analysis and nanodesign. Nucleic Acids Res. 2008; 36:D392-D397. [PubMed: 17947325]

38. Wagner C, Ehresmann C, Ehresmann B, Brunel C. Mechanism of dimerization of bicoid mRNA: initiation and stabilization. J Biol Chem. 2004; 279:4560-4569. [PubMed: 14607826]

39. Chen C, Sheng S, Shao Z, Guo P. A dimer as a building block in assembling RNA: A hexamer that gears bacterial virus phi29 DNA-translocating machinery. J Biol Chem. 2000; 275(23):17510 17516. [PubMed: 10748150]

40. Ponchon L, Beauvais G, Nonin-Lecomte S, Dardel F. A generic protocol for the expression and purification of recombinant RNA in Escherichia coli using a tRNA scaffold. Nat Protoc. 2009; 4:947-959. [PubMed: 19478810] 
41. Kuwabara T, et al. Formation of a catalytically active dimer by tRNA-driven short ribozymes. Nature Biotechnology. 1998; 16:961-965.

42. Fire A, et al. Potent and specific genetic interference by double-stranded RNA in Caenorhabditis elegans. Nature. 1998; 391:806-811. [PubMed: 9486653]

43. Li H, Li WX, Ding SW. Induction and suppression of RNA silencing by an animal virus. Science. 2002; 296:1319-1321. [PubMed: 12016316]

44. Breaker RR. Complex riboswitches. Science. 2008; 319:1795-1797. [PubMed: 18369140]

45. Fabian MR, Sonenberg N, Filipowicz W. Regulation of mRNA translation and stability by microRNAs. Annu Rev Biochem. 2010; 79:351-379. [PubMed: 20533884]

46. Zhang C. Novel functions for small RNA molecules. Curr Opin Mol Ther. 2009; 11:641-651. [PubMed: 20072941]

47. Marvin MC, Engelke DR. Broadening the mission of an RNA enzyme. J Cell Biochem. 2009; 108:1244-1251. [PubMed: 19844921]

48. Benenson Y. RNA-based computation in live cells. Curr Opin Biotechnol. 2009; 20:471-478. [PubMed: 19720518]

49. Shlyakhtenko LS, et al. Silatrane-based surface chemistry for immobilization of DNA, proteinDNA complexes and other biological materials. Ultramicroscopy. 2003; 97:279-287. [PubMed: 12801681]

50. Guo S, Tschammer N, Mohammed S, Guo P. Specific delivery of therapeutic RNAs to cancer cells via the dimerization mechanism of phi29 motor pRNA. Hum Gene Ther. 2005; 16:1097-1109. [PubMed: 16149908]

51. Khaled A, Guo S, Li F, Guo P. Controllable Self-Assembly of Nanoparticles for Specific Delivery of Multiple Therapeutic Molecules to Cancer Cells Using RNA Nanotechnology. Nano Letters. 2005; 5:1797-1808. [PubMed: 16159227]

52. Shu D, Huang L, Hoeprich S, Guo P. Construction of phi29 DNA-packaging RNA (pRNA) monomers, dimers and trimers with variable sizes and shapes as potential parts for nano-devices. $\mathrm{J}$ Nanosci Nanotechnol. 2003; 3:295-302. [PubMed: 14598442]

53. Turner R, Tijan R. Leucine repeats and an adjacent DNA binding domain mediate the formation of functional c-Fos and c-Jun heterodimers. Science. 1989; 243:1689-1694. [PubMed: 2494701]

54. Liu D, et al. Tensegrity: construction of rigid DNA triangles with flexible four-arm DNA junctions. J Am Chem Soc. 2004; 126:2324-2325. [PubMed: 14982434]

55. Rothemund PW, Papadakis N, Winfree E. Algorithmic self-assembly of DNA Sierpinski triangles. PLoS Biol. 2004; 2:e424. [PubMed: 15583715]

56. Park SH, et al. Three-helix bundle DNA tiles self-assemble into 2D lattice or 1D templates for silver nanowires. Nano Lett. 2005; 5:693-696. [PubMed: 15826110]

57. Weizmann Y, et al. A polycatenated DNA scaffold for the one-step assembly of hierarchical nanostructures. Proc Natl Acad Sci U S A. 2008; 105:5289-5294. [PubMed: 18391204]

58. Aldaye FA, Sleiman HF. Modular access to structurally switchable 3D discrete DNA assemblies. J Am Chem Soc. 2007; 129:13376-13377. [PubMed: 17939666]

59. Zheng J, et al. From molecular to macroscopic via the rational design of a self-assembled 3D DNA crystal. Nature. 2009; 461:74-77. [PubMed: 19727196]

60. Rothemund PWK. Folding DNA to create nanoscale shapes and patterns. Nature. 2006; 440:297302. [PubMed: 16541064]

61. Andersen ES, et al. Self-assembly of a nanoscale DNA box with a controllable lid. Nature. 2009; 459:73-76. [PubMed: 19424153]

62. Ke YG, et al. Self-assembled water-soluble nucleic acid probe tiles for label-free RNA hybridization assays. Science. 2008; 319:180-183. [PubMed: 18187649]

63. Douglas SM, Chou JJ, Shih WM. DNA-nanotube-induced alignment of membrane proteins for NMR structure determination. Proc Natl Acad Sci U S A. 2007; 104:6644-6648. [PubMed: 17404217]

64. Endo M, Seeman NC, Majima T. DNA tube structures controlled by a four-way-branched DNA connector. Angew Chem Int Ed Engl. 2005; 44:6074-6077. [PubMed: 16124026] 
65. Tanaka K, et al. A discrete self-assembled metal array in artificial DNA. Science. 2003; 299:12121213. [PubMed: 12595687]

66. Yurke B, et al. A DNA-fuelled molecular machine made of DNA. Nature. 2000; 406:605-608. [PubMed: 10949296]

67. Lin C, et al. In vivo cloning of artificial DNA nanostructures. Proc Natl Acad Sci U S A. 2008; 105:17626-17631. [PubMed: 18927233]

68. Eckardt LH, et al. DNA nanotechnology: Chemical copying of connectivity. Nature. 2002; 420:286. [PubMed: 12447432]

69. Endo M, et al. Programmed-assembly system using DNA jigsaw pieces. Chemistry. 2010; 16:5362-5368. [PubMed: 20391568]

70. Dietz H, Douglas SM, Shih WM. Folding DNA into twisted and curved nanoscale shapes. Science. 2009; 325:725-730. [PubMed: 19661424]

71. Cherny DI, Eperon IC, Bagshaw CR. Probing complexes with single fluorophores: factors contributing to dispersion of FRET in DNA/RNA duplexes. Eur Biophys J. 2009; 38:395-405. [PubMed: 19015840]

72. Shapiro BA. Computational Design Strategies for RNA Nanostructures. J Biomol Str Dyn. 2009; $26: 820$.

73. Yingling YG, Shapiro BA. Computational design of an RNA hexagonal nanoring and an RNA nanotube. Nano Letters. 2007; 7:2328-2334. [PubMed: 17616164]

74. Zuker M. Mfold web server for nucleic acid folding and hybridization prediction. Nucleic Acids Res. 2003; 31:3406-3415. [PubMed: 12824337]

75. Markham NR, Zuker M. UNAFold: soft ware for nucleic a cid folding and hybridization. Methods Mol Biol. 2008; 453:3-31. [PubMed: 18712296]

76. Bindewald E, et al. Computational strategies for the automated design of RNA nanoscale structures from building blocks using NanoTiler. Journal of Molecular Graphics \& Modelling. 2008; 27:299_ 308. [PubMed: 18838281]

77. Afonin KA, et al. In vitro assembly of cubic RNA-based scaffolds designed in silico. Nat Nanotechnol. 2010; 5:676-682. [PubMed: 20802494]

78. Chakraborty S, Modi S, Krishnan Y. The RNA2-PNA2 hybrid i-motif-a novel RNA-based building block. Chem Commun (Camb ). 2008:70-72. [PubMed: 18401893]

79. Afonin KA, Cieply DJ, Leontis NB. Specific RNA self-assembly with minimal paranemic motifs. J Am Chem Soc. 2008; 130:93-102. [PubMed: 18072767]

80. Lescoute A, Westhof E. Topology of three-way junctions in fo lded RNAs. RNA. 2006; 12:83-93. [PubMed: 16373494]

81. Ouellet J, et al. Structure of the three-way helical junction of the hepatitis C virus IRES element. RNA. 2010; 16:1597-1609. [PubMed: 20581129]

82. dela PM, Dufour D, Gallego J. Three- way RNA junctions with remote tertiary contacts: a recurrent and highly versatile fold. RNA. 2009; 15:1949-1964. [PubMed: 19741022]

83. Griffiths-Jones $\mathrm{S}$, et al. Rfam: annotating non-coding RNAs in complete genomes. Nucleic Acids Res. 2005; 33:D121-D124. [PubMed: 15608160]

84. Abraham M, Dror O, Nussinov R, Wolfson HJ. Analysis and classification of RNA tertiary structures. RNA. 2008; 14:2274-2289. [PubMed: 18824509]

85. Guo P, Erickson S, Anderson D. A small viral RNA is required for in vitro packaging of bacteriophage phi29 DNA. Science. 1987; 236:690-694. [PubMed: 3107124]

86. Xiao F, Demeler B, Guo P. Assembly Mechanism of the Sixty-Subunit Nanoparticles via Interaction of RNA with the Reengineered Protein Connector of phi29 DNA-Packaging Motor. ACS Nano. 2010; 4:3293-3301. [PubMed: 20509670]

87. Shu D, Zhang H, Jin J, Guo P. Counting of six p RNAs of phi29 DNA-packaging motor with customized single molecule dual-view system. EMBO J. 2007; 26:527-537. [PubMed: 17245435]

88. Woodson SA. Compact intermediates in RNA folding. Annu Rev Biophys. 2010; 39:61-77. [PubMed: 20192764] 
89. Gugliotti LA, Feldheim DL, Eaton BE. RNA-mediated metal-metal bond formation in the synthesis of hexagonal palladium nanoparticles. Science. 2004; 304:850-852. [PubMed: 15087507]

90. Koyfman AY, et al. Controlled spacing of cationic gold nanoparticles by nanocrown RNA. J Am Chem Soc. 2005; 127:11886-11887. [PubMed: 16117496]

91. Oguro A, Ohtsu T, Nakamura Y. An aptamer-based biosensor for mammalian initiation factor eukaryotic initiation factor 4A. Analyt Biochem. 2009; 388:102-107. [PubMed: 19250914]

92. Mi J, et al. In vivo selection of tumor-targeting RNA motifs. Nature Chemical Biology. 2010; 6:22-24.

93. Liu Y, et al. Targeting hypoxia-inducible factor-1alpha with Tf-PEI-shRNA complex via transferrin receptor-mediated endocytosis inhibits melanoma growth. Mol Ther. 2009; 17:269_ 277. [PubMed: 19066596]

94. Kumar P, et al. Transvascular delivery of small interfering RNA to the central nervous system. Nature. 2007; 448:39-43. [PubMed: 17572664]

95. Kruger K, et al. Self-splicing RNA: autoexcision and autocyclization of the ribosomal RNA intervening sequence of Tetrahymena. Cell. 1982; 31:147-157. [PubMed: 6297745]

96. Guerrier-Takada C, et al. The RNA moiety of ribonuclease P is the catalytic subunit of the enzyme. Cell. 1983; 35:849-857. [PubMed: 6197186]

97. Ellington AD, Szostak JW. In vitro selection of RNA molecules t hat bind specific ligands. Nature. 1990; 346:818-822. [PubMed: 1697402]

98. Tuerk C, Gold L. Systematic evolution of ligands by exponential enrichment: RNA ligands to bacteriophage T4 DNA ploymerase. Science. 1990; 249:505-510. [PubMed: 2200121]

99. Ellington AD. Back to the future of nucleic acid self-amplification. Nature Chemical Biology. 2009; 5:200-201.

100. Zhou J, Li H, Zaia J, Rossi JJ. Novel dual inhibitory function aptamer-siRNA delivery system for HIV-1 therapy. Mol Ther. 2008; 16:1481-1489. [PubMed: 18461053]

101. Bunka DH, et al. Production and characterization of RNA aptamers specific for amyloid fibril epitopes. J Biol Chem. 2007; 282:34500-34509. [PubMed: 17878167]

102. Sudarsan N, et al. Riboswitches in eubacteria sense the second messenger cyclic di-GMP. Science. 2008; 321:411-413. [PubMed: 18635805]

103. Ogawa A, Maeda M. An artificial aptazyme-based riboswitch and its cascading system in Ecoli . Chembiochem. 2008; 9:206-209. [PubMed: 18098257]

104. Shahbabian K, Jamalli A, Zig L, Putzer H. RNase Y, a novel endoribonuclease, initiates riboswitch turnover in Bacillus subtilis. EMBO J. 2009; 28:3523-3533. [PubMed: 19779461]

105. Prabha S, Zhou WZ, Panyam J, Labhasetwar V. Size-dependency of nanoparticle-mediated gene transfection: studies with fractionated nanoparticles. Int J Pharm. 2002; 244:105-115. [PubMed: 12204570]

106. Guo S, Huang F, Guo P. Construction of folate- conjugated pRNA of bacteriophage phi29 DNA packaging motor for delivery of chimeric siRNA to nasopharyngeal carcinoma cells. Gene Ther. 2006; 13:814-820. [PubMed: 16482206]

107. Huifang M, Zhang, et al. Target delivery of anti-coxsachievirus siRNAs using ligand-conjugated packaging RNAs. Antiviral Res. 2009; 83(3):307-316. [PubMed: 19616030]

108. Watts JK, Deleavey GF, Damha MJ. Chemically modified siRNA: tools and applications. Drug Discovery Today. 2008; 13:842-855. [PubMed: 18614389]

109. Madhuri V, Kumar VA. Design,synthesis and DNA/RNA binding studies of nucleic acids comprising stereoregular and acyclic polycarbamate backbone: polycarbamate nucleic acids (PCNA). Org Biomol Chem. 2010 [Epub ahead of print].

110. Mathe C, Perigaud C. Recent approaches in the synthesis of conformationally restricted nucleoside analogues. European Journal of Organic Chemistry. 2008:1489-1505.

111. Patra A, Richert C. High fidelity base pairing at the 3'-terminus. J Am Chem Soc. 2009; 131:12671-12681. [PubMed: 19722718]

112. Efimov VA, Fediunin SV, Chakhmakhcheva OG. Cross-linked nucleic acids: formation, structure, and biological function. Bioorg Khim. 2010; 36:56-80. [PubMed: 20386579] 
113. Song Z, et al. Synthesis and oxidation-induced DNA cross-linking capabilities of bis(catechol) quaternary ammonium derivatives. Chemistry. 2008; 14:5751-5754. [PubMed: 18491338]

114. Stengel G, Urban M, Purse BW, Kuchta RD. Incorporation of the fluorescent ribonucleotide analogue tCTP by T7 RNA polymerase. Anal Chem. 2010; 82:1082-1089. [PubMed: 20067253]

115. Solomatin S, Herschlag D. Methods of Site-Specific Labeling of RNA with Fluorescent Dyes. Methods in Enzymology, Vol 469: Biophysical, Chemical, and Functional Probes of Rna Structure, Interactions and Folding, Pt B. 2009; 469:47-68.

116. Lavergne T, Bertrand JR, Vasseur JJ, Debart F. A base-labile group for 2'-OH protection of rib onucleosides: a major challenge for RNA synthesis. Chemistry. 2008; 14:9135-9138. [PubMed: 18767078]

117. Hoeprich S, Guo P. Computer modeling of three-dimensional structure of DNA-packaging RNA(pRNA) monomer, dimer, and hexamer of phi29 DNA Packaging motor. J Biol Chem. 2002; 277(23):20794-20803. [PubMed: 11886855] 


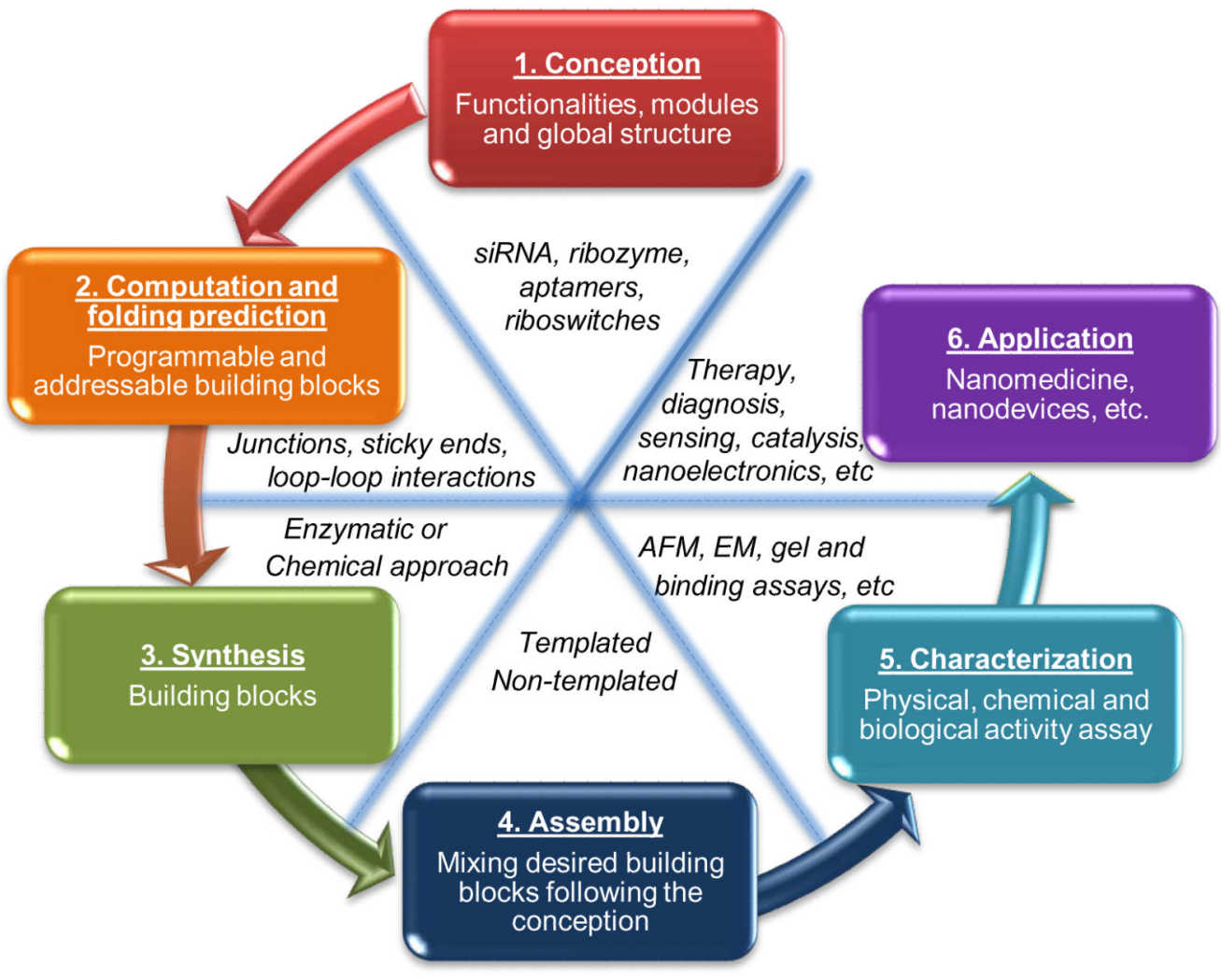

Figure 1. Approaches in RNA Nanotechnology

The construction of RNA nanoparticles involves several steps: Following the conception, a computational approach can be applied to predict the folding and structure of the building blocks as well as the consequences of inter-RNA interactions in RNA nanoparticle assembly. After the synthesis of monomeric building blocks, the individual subunits can be further assembled into quaternary architectures utilizing the spontaneous self-folding property of RNA. The assembled RNA nanostructures will be characterized to ensure proper folding with desired structural/functional capabilities. After thorough evaluation, the nanoparticles will be then be used for various applications. 


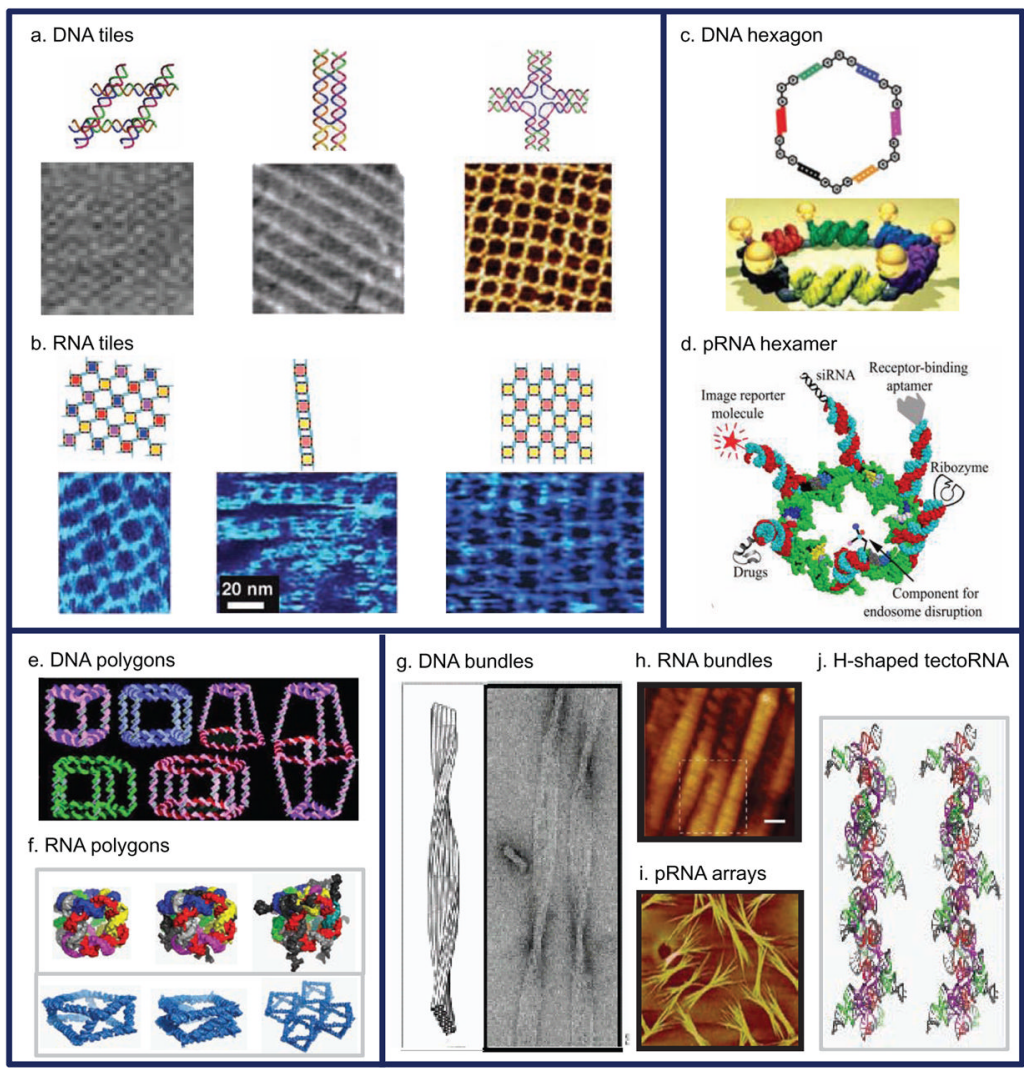

Figure 2. Comparison of self-assembled DNA (a,c,e,g), and RNA (b,d,f,h,I,j) nanoparticles Images of DNA tiles (a) ${ }^{1,2}$ and RNA tiles via tectosquares (b) ${ }^{13}$; illustration of hexameric DNA gold nanoparticle (c) $)^{1,2}$, pRNA hexameric ring (d) ${ }^{50,87}$; DNA 3D polygons $(\mathbf{e})^{2}$; and RNA cubic scaffolds (f) ${ }^{30,77}$; images of DNA bundles $(\mathbf{g})^{70}$, RNA bundles (h) ${ }^{23}$, pRNA arrays (i) ${ }^{12}$; and 3D model of H-shaped tectoRNA $(\mathbf{j})^{21}$. All images are taken by AFM except (g) and (j), as well as the first two images of (a), which are TEM images. All images were adapted from the individual references with permission. 

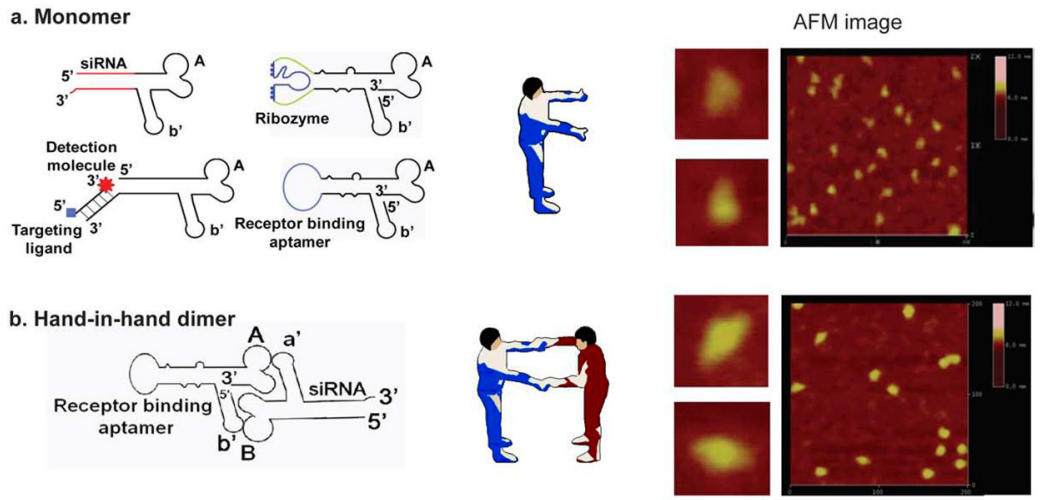

c. Trimer
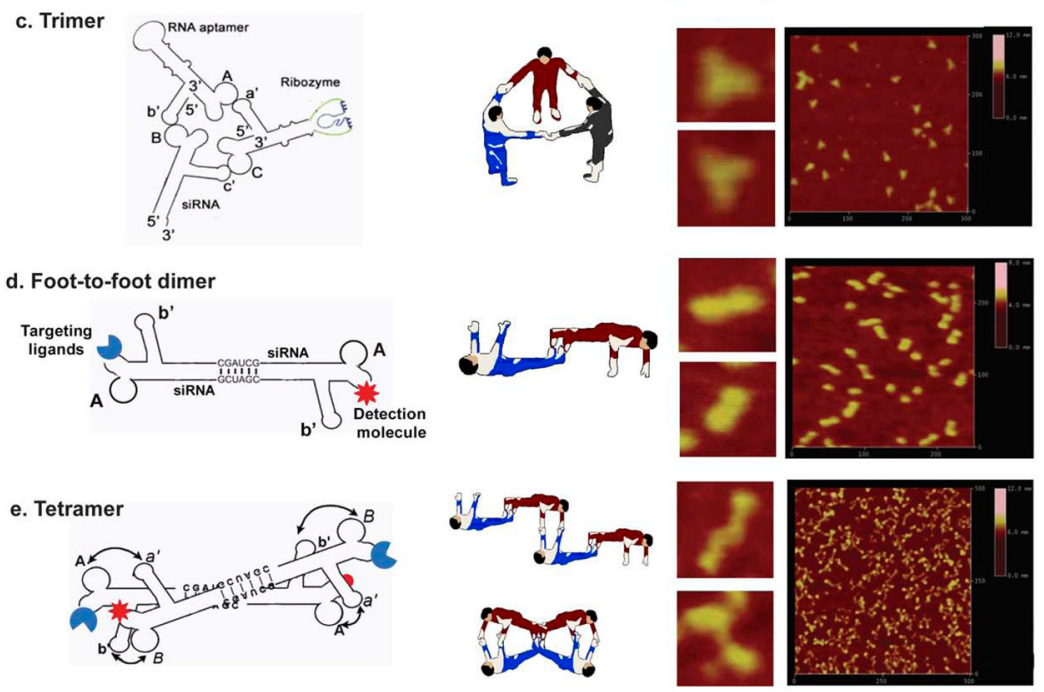

Figure 3. Applications of RNA nanotechnology

Therapeutic nanoparticles harboring siRNA, ribozyme, aptamer, and other moieties are constructed using bacteriophage phi29 pRNA left- and right-hand interlocking loops or palindrome sequence without template ${ }^{8,12,39}$. Uppercase and lowercase letters signify right and left hand (a). Same letter pair, e.g., A-a' indicates complementarity ${ }^{28}$. Dimers assemble via pRNA A- $\mathrm{b}^{\prime}$ and $\mathrm{B}-\mathrm{a}^{\prime}(\mathbf{b})$. Trimers form using pRNA A- $\mathrm{b}^{\prime}, \mathrm{B}-\mathrm{c}^{\prime}$ and $\mathrm{C}-\mathrm{a}^{\prime}(\mathbf{c})$. Foot-tofoot dimers form via end Palindrome sequence (d). Tetramers assemble by the combination of interlocking loops and palindrome mechanism (e). The right panel depicts AFM images adapted from ${ }^{12,39}$ with permission. 
Table 1

Differences between DNA and RNA

\begin{tabular}{|c|c|c|c|}
\hline & & DNA & RNA \\
\hline \multicolumn{2}{|l|}{ Elements } & 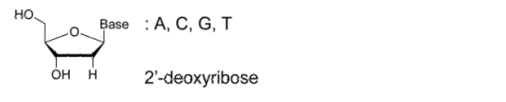 & ${ }_{\mathrm{OH}}^{\mathrm{HO}} \mathrm{OH}_{\text {ribose }}^{\text {Base }}: \mathrm{A}, \mathrm{C}, \mathrm{G}, \mathrm{U}$ \\
\hline \multicolumn{2}{|l|}{ Base pairing } & Canonical Watson-Crick (W-C) & Canonical and Non-canonical W-C \\
\hline \multicolumn{2}{|l|}{ Acidic effect } & $\begin{array}{l}\text { Depurination: Apurine DNA sensitive to } \\
\text { cleavage }\end{array}$ & Stable \\
\hline \multicolumn{2}{|l|}{ Alkaline effect } & Stable up to $\mathrm{pH} 12$ & Sensitive to alkaline hydrolysis \\
\hline \multicolumn{2}{|l|}{ Configuration } & $\begin{array}{l}\text { Predominantly B form: } \\
\text { - } \quad \text { Base pairs/turn of the helix: } 10.5 ; \\
\text { - } \\
\text { - } \\
\text { Pitch: } 3.5 \mathrm{~nm} ; \\
\text { - Helix rise/bp: } 0.314 \mathrm{~nm} ; \\
\text { Humidity: Nucleotide: } \mathrm{H}_{2} \mathrm{O}=1: 1\end{array}$ & $\begin{array}{cl}\text { A form: } & \\
\text { - } & \text { Base pairs/turn of the helix: 10.9; } \\
\text { - } & \text { Pitch: } 2.5 \mathrm{~nm} ; \\
\text { - } & \text { Helix rise/bp: } 0.275 \mathrm{~nm} ; \\
\text { - } & \text { Humidity: Nucleotide: } \mathrm{H}_{2} \mathrm{O}=1: 0.7\end{array}$ \\
\hline \multicolumn{2}{|l|}{ Chemical Stability } & Relatively stable but sensitive to DNase & $\begin{array}{l}\text { Unstable, sensitive to RNase, but stable after chemical } \\
\text { modification, e.g. } 2^{\prime}-\mathrm{F} \text { or } 2^{\prime} \text {-OMe modification }\end{array}$ \\
\hline \multicolumn{2}{|l|}{ Thermal stability } & G:C more stable than A:T & $\begin{array}{l}\text { Thermally more stable than DNA, especially for RNA } \\
\text { motifs and modules with particular bends or stacks }\end{array}$ \\
\hline \multicolumn{2}{|l|}{ Free energy, $\Delta \mathrm{G}^{\mathrm{o}}$} & $-1.4^{-1} \mathrm{KJ} . \mathrm{mol}$ per bp stack 25 & -3.6 to $-8.5 \mathrm{KJ} \cdot \mathrm{mol}^{-1}$ per bp stack 25 \\
\hline \multicolumn{2}{|l|}{ Helix formation } & Need minimum 4 nucleotides & Need minimum 2 nucleotides 26,27 \\
\hline \multicolumn{2}{|c|}{ Intermolecular interactions } & Cohesive ends, crossover motifs & $\begin{array}{l}\text { Cohesive ends, crossover motifs, Kissing loops, } \\
\text { interlocking loops }\end{array}$ \\
\hline \multirow{2}{*}{ In vivo replication } & Initiation & Origin of replication with primer & Promoter, exact nucleotide to start without primer \\
\hline & Termination & No nature sequence for replication termination. & Specific transcription terminators. \\
\hline \multirow{2}{*}{ In vitro synthesis } & Enzymatic & DNA polymerase, $\mathrm{PCR}$. & T7/SP6 Transcription. \\
\hline & Chemical & Up to 160 nucleotides; Low cost & Up to 117 nucleotide; High cost and low yield \\
\hline
\end{tabular}


Table 2

Techniques for constructing RNA nanoparticles

\begin{tabular}{|c|c|c|c|}
\hline \multicolumn{4}{|r|}{ - } \\
\hline Tactic 1 & $\begin{array}{c}1 \\
\text { Tactic } 2 \\
\end{array}$ & $\begin{array}{c}1 \\
\text { Tactic } 3 \\
\end{array}$ & Tactic 4 \\
\hline $\begin{array}{l}\text { Utilize the assembly } \\
\text { mechanism of natural }\end{array}$ & $\begin{array}{l}\text { Import principle of DNA } \\
\text { nanotechnology }\end{array}$ & $\begin{array}{l}\text { Apply computational } \\
\text { methods }\end{array}$ & $\begin{array}{l}\text { Utilize existing RNA } \\
\text { structure as building blocks }\end{array}$ \\
\hline $\begin{array}{l}\text { RNA motifs } \\
\text { - Retrovirus kissing loop } \\
\text { - Phi29 pRNA } \\
\text { - Bicoid mRNA }\end{array}$ & $\begin{array}{l}\text { - Junctions and branches } \\
\text { - Jigsaw puzzles } \\
\text { - Bundle, twisted bundles } \\
\text { - Cubic scaffolds }\end{array}$ & $\begin{array}{l}\text { Two steps: } \\
\text { - define building block } \\
\text { - self-assembly of the RNA } \\
\text { into quaternary architectures }\end{array}$ & $\begin{array}{l}\text { - Phi29 pRNA dimer, trimer, } \\
\text { tetramer, hexamer and arrays } \\
\text { - RNA architectonics } \\
\text { - Junctions and branches } \\
\text { - Ribozyme ligase to assemble } \\
\text { RNA motifs } \\
\text { - Palindrome sequence }\end{array}$ \\
\hline \multicolumn{4}{|c|}{ Fusion or conjugation with } \\
\hline & Riboswitch & Ribozyme & miRNA \\
\hline
\end{tabular}

